\title{
Model of Grey Mean Generating Function and Its Application Pesticide Quantity Prediction
}

\author{
Xiu-Hong ZHANG ${ }^{1, a}$, Yong LIN $^{2 *, b}$ \\ ${ }^{1}$ School of Information Science and Technology, Hainan Normal University ,Haikou, Hainan, China \\ ${ }^{2}$ Chinese Academy of Tropical Agricultural Sciences Institute of Environment and Plant Protection \\ Haikou, Hainan, China *Corresponding author \\ a443635106@qq.com, b1171101509@qq.com
}

Keywords: Pesticide Quantity; prediction model; Mean generating function.

\begin{abstract}
Now pesticide spray is an effective means to ensure and eliminate crop pests and diseases increased yield, but also impact on the ecological environment, food security, and so prediction of the amount of application of pesticide is an important topic in the current research. Therefore, this paper puts forward a kind of with the quantity of application of pesticide for prediction of grey function combination forecasting model. It can successfully predicate the sample data, and the method has good feasibility.
\end{abstract}

\section{Introduction}

Pesticide quantity forecast refers to forecasting the amount of pesticide dosage in the future-time acording to known years based on the prediction model. It is great significance for agricultural production and environmental protection. Because of predicted results is influenced by known conditions or influenced by the unknown conditions, so the factors and hierarchical structure of pesticide quantity forecast model is complex. The result of pesticide quantity forecast is influenced by external factors (cost, using the environment) and the system of internal factors (such as toxicity and dosage form) ,but its change is not clear. Because the output of the medication time sequence is the result of the interaction between various factors, we can find the regularity of them.

This paper proposes a combination of mean generating function combination of grey prediction model to predict the medication pesticides. Mainly based on the gray GM $(1,1)$ model to reveal the development trend, combined with the mean generating function model for data variation rule, combination of combination forecast, eliminate the error of the respective, complementary advantages, this article through to the cities and counties of Hainan province each year as samples for the latest years of applying pesticide content seems to build model to forecast, compared with the real value verify its feasibility and effectiveness of verify the feasibility and effectiveness of the model.

\section{The prediction theory of grey mean generating function}

The ideas of the two kinds of forecast ways combination forecast is put forward by Bates and Granger. The main idea is to turn to each kind of model prediction results, as a new prediction samples according to eliminate the original forecasts new prediction error, so as to dispersion prediction error of the impact of various methods and increase the prediction precision.

Grey GM(1,1) model. Grey system theory put forward by professor Deng Julong ${ }^{[7]}$. Its mainly characteristic is that reducing the sample data randomness by applying statistical data generation methods such as accumulative, standardization method etc, and to reveal its own regularity gradually, and make its effectively used in model, on the premise of save all the data original features.

The GM $(1,1)$ model is applicable to reveal the general trend of development and change of forecasted object. The process of grey prediction model GM $(1,1)$ as follows:(1) Determine the original time series. (2) Generate the first-order accumulative sequence. (3) Estimate parameter vector by least squares. (4) Build discrete response equation. (5) Continuous subtraction restore sequence. (6) Get prediction results. 
Mean generating function model. Mean generating function model is a new prediction model derived from arithmetic mean by Feng-ying Wei .Its principle is that build and develop phase space and establish a periodic function via the relevance and memory properties of the system state before and after. Looking for periodic trends and establish the corresponding statistical prediction model via analyze and statistic the original data sequence. Studies have shown that this method possesses strong ability of fitting and forecasting for the extremum of the wave nature of the data. It can accurately extract the forecast object law of cyclical fluctuations. The forecast model of Mean generating function builds process as follows: (1) Standardization of original data. (2) Generate the mean generating function. (3)Mean generating function cycle extension matrix. (4)Main constituent analysis extension matrix. (5) Main component model is put forward. (6) Get prediction results.

\section{Build pesticide model to predict the medication}

This paper build a new model to predict amount of pesticide base on grey prediction model with strong prediction ability and overall trends and predict stability of mean generating function mode. This model mainly divides the original data sequence $\left\{S_{t}\right\}$ into two parts: trend term $\left\{M_{t}\right\}$ and Random fluctuations item $\left\{e_{t}\right\}$.

$$
S_{t}=M_{t}+e_{t}
$$

Trend item $\left\{\mathrm{M}_{\mathrm{t}}\right\}$ is for the grey model prediction, Random fluctuations item $\left\{e_{t}\right\}$ is for the mean generating function mode prediction. Two kinds of model prediction are summed up with can complete the medication to predict pesticides. The main steps are as follows:

1.Establishing the original time series with amount of pesticide of every counties in Hainan in over the years $x^{(0)}=\left\{x^{(0)}\{1\}, x^{(0)}\{2\}, \ldots, x^{(0)}\{n\}\right\}$, and accumulation process the time sequence in turn. Build an albino differential equation

$$
\begin{gathered}
x_{k}^{(1)}=\sum_{j=1}^{k} x_{j}^{(0)}, k=1,2, \cdots, n \\
\frac{d x^{(1)}}{d t}+a x^{(1)}=u
\end{gathered}
$$

a,u is the middle variables for solving, $\hat{a}=[a, u]^{T}, y_{n}=\left[x_{2}^{(0)}, x_{3}^{(0)}, \cdots, x_{n}^{(0)}\right]^{T}$ is parameter vector. 2.Building a matrix $\mathrm{B}$, estimating and solving $\widehat{a}$ using the least squares estimate method and establish the corresponding discrete equations.

$$
\begin{gathered}
B=\left[\begin{array}{cc}
-\left(x_{2}^{(1)}+x_{1}^{(1)}\right) / 2 & 1 \\
-\left(x_{2}^{(1)}+x_{1}^{(1)}\right) / 2 & 1 \\
\cdots & \cdots \\
-\left(x_{2}^{(1)}+x_{1}^{(1)}\right) / 2 & 1
\end{array}\right] \\
\widehat{a}=\left(B^{T} B\right)^{-1} B^{T} y_{n} \\
\widehat{x}_{k+1}^{(1)}=\left[x_{1}^{(1)}-\frac{u}{a}\right] e^{-a k}+\frac{u}{a}, k=0,1, \cdots, n-1 \\
\widehat{x}_{k+1}^{(0)}=\widehat{x}_{k+1}^{(1)}-\widehat{x}_{k}^{(1)} \quad k=0,1, \cdots, n-1
\end{gathered}
$$

$\widehat{x}_{k+1}^{(0)}$ is the trend value of the original data $x_{i}^{(0)}$ extract from $\operatorname{GM}(1,1)$ model.

3. Define the relative results of pesticides usage amount estimated of time series prediction $z(i)=x_{i}^{(0)}-\widehat{x}_{i}^{(0)} \quad i=1, \cdots, n$. Build the grey relative sequence $\{Z(1), Z(2), \ldots, Z(n)\}$ and define the mean generating function.

$$
\begin{gathered}
\bar{z}_{l}(i)=\frac{1}{n_{i}} \sum_{j=0}^{n_{i}-1} z(i+j l) \quad(i=1,2, \cdots, l, 1 \leq l \leq m) \\
n_{l}=I N T(n / l), m \leqslant I N T(n / 2), \quad \text { INT said is rounded }
\end{gathered}
$$


4. Base on (8), mean generating function will be requested to take periodic extension and constitute extension matrix F.

$$
F_{n \times l}=\left[\begin{array}{ccccc}
\bar{z} & \bar{z}_{2}(1) & \bar{z}_{3}(1) & \cdots & \bar{z}_{m}(1) \\
\bar{z} & \bar{z}_{2}(2) & \bar{z}_{3}(2) & \cdots & \bar{z}_{m}(2) \\
\bar{z} & \bar{z}_{2}(1) & \bar{z}_{3}(3) & \cdots & \bar{z}_{m}(3) \\
\bar{z} & \bar{z}_{2}(2) & \bar{z}_{3}(1) & \cdots & \bar{z}_{m}(4) \\
\cdots & \cdots & \cdots & \cdots & \cdots \\
\bar{z} & \bar{z}_{2}\left(i_{2}\right) & \bar{z}_{3}\left(i_{3}\right) & \cdots & \bar{z}_{3}\left(i_{m}\right)
\end{array}\right]
$$

5.Analysing the principal component of the extension matrix , and calculating the covariance matrixion $\mathrm{S}$ according to the covariance formula.

$$
s_{i j}=\frac{1}{n} \sum_{k=1}^{n}\left(x_{i k}-\bar{x}_{i}\right)\left(x_{j k}-\bar{x}_{j}\right)
$$

6.Calculated the ratio of the characteristic values before number $\mathrm{m}$ and the sum of characteristic values $\rho$. The threshold value u can be selected according to specific circumstances.

$$
\rho=\frac{\sum_{i=1}^{m} \lambda_{i}}{\sum_{j=1}^{1} \lambda_{j}} \geq u
$$

7.build forecasting model.

$$
Z_{n \times 1}=V_{n \times m} \Phi_{m \times l}^{\prime}+\xi_{n \times l}^{\prime}
$$

$V_{n \times m}$ is principal component matrix, $\xi$ is error, $\Phi_{m \times l}^{\prime}$ is regression coefficient.

$$
\Phi_{m \times l}^{\prime}=\left(V_{m \times n}^{T} V_{n \times m}\right)^{-1} V_{m \times n}^{T} Z_{n \times l}
$$

8.Get I the fitting value at $n$ time and the predictive value at $n+1$ time.

$$
\begin{aligned}
& Z_{n \times l}=F_{n \times l} \Phi_{l \times 1}+\xi_{n \times l} \\
& V_{n \times m}=F_{n \times l} C_{l \times m}^{T} \\
& F_{n \times l}=V_{n \times m} C_{n \times l} \\
& \Phi_{l \times l}=C_{l \times m}^{T} \Phi_{m \times l}^{\prime} \\
& \widehat{z}(n)=\sum_{j=1}^{l} f_{n, j} \times \Phi(j) \\
& \widehat{z}(n+1)=\sum_{j=1}^{l} f_{n+1, j} \times \Phi(j)
\end{aligned}
$$

\section{Sample calculation}

Hainan province pesticide statistics of every counties as shown in table 1. Taking the groups of data before 12 as a sample,and predicting the groups of data13 and 14, so as to verify this model effectiveness. To make the sequence of 18 counties in Hainan as $\mathrm{X}_{1}-\mathrm{X} 1_{8}$,sum is $\mathrm{X}_{19}$, time series is as for $\mathrm{T}_{1}-\mathrm{T}_{14}$. The sample as shown table1.

Table 1 Hainan every counties pesticide amount time series sample

\begin{tabular}{cccccccc}
\hline & $\mathrm{T} 1$ & $\mathrm{~T} 2$ & $\mathrm{~T} 3$ & $\ldots$ & $\mathrm{T} 12$ & $\mathrm{~T} 13$ & $\mathrm{~T} 14$ \\
$\mathrm{X} 1$ & 64 & 76 & 76 & $\ldots$ & 1198 & 1616 & 1425 \\
$\mathrm{X} 2$ & 337 & 291 & 317 & $\ldots$ & 1345 & 2942 & 3649 \\
$\mathrm{X} 3$ & 18 & 18 & 19 & $\ldots$ & 155 & 166 & 620 \\
$\mathrm{X} 4$ & 716 & 691 & 1666 & $\ldots$ & 2447 & 2321 & 1456 \\
$\mathrm{X} 5$ & 638 & 580 & 714 & $\ldots$ & 2188 & 1915 & 1856 \\
$\ldots$ & $\ldots$ & $\ldots$ & $\ldots$ & $\ldots$ & $\ldots$ & $\ldots$ & $\ldots$ \\
$\mathrm{X} 17$ & 156 & 202 & 223 & $\ldots$ & 1006 & 1308 & 1212 \\
$\mathrm{X} 18$ & 103 & 93 & 105 & $\ldots$ & 145 & 140 & 156 \\
$\mathrm{X} 19$ & 12563 & 10963 & 13573 & $\ldots$ & 39637 & 43479 & 39930 \\
\hline
\end{tabular}


According to the model as described above, The prediction result of pesticide amount is shown as table 2 .

Table 2 The prediction result of pesticide amount

\begin{tabular}{ccccc}
\hline time series & set & predicted value & actual value & relative error \\
\hline & $\mathrm{X}_{1}$ & 1529 & 1616 & $5.38 \%$ \\
$\mathrm{X}_{2}$ & 2745 & 2942 & $6.70 \%$ \\
& $\mathrm{X}_{3}$ & 158 & 166 & $4.82 \%$ \\
& $\mathrm{X}_{4}$ & 2212 & 2321 & $4.70 \%$ \\
$\mathrm{~T}_{13}$ & $\mathrm{X}_{5}$ & 1787 & 1915 & $6.68 \%$ \\
& $\cdots$ & $\ldots$ & $\ldots$ & $\ldots$ \\
& $\mathrm{X}_{16}$ & 789 & 753 & $4.78 \%$ \\
& $\mathrm{X}_{17}$ & 1423 & 1308 & $8.79 \%$ \\
& $\mathrm{X}_{18}$ & 135 & 140 & $3.57 \%$ \\
& $\mathrm{X}_{1}$ & 1302 & 1425 & $8.63 \%$ \\
& $\mathrm{X}_{2}$ & 3440 & 3649 & $5.73 \%$ \\
& $\mathrm{X}_{3}$ & 580 & 620 & $6.45 \%$ \\
& $\cdots$ & $\cdots$ & $\cdots$ & $\cdots$ \\
& $\mathrm{X}_{16}$ & 837 & 793 & $5.55 \%$ \\
& $\mathrm{X}_{17}$ & 1302 & 1212 & $7.43 \%$ \\
& $\mathrm{X}_{18}$ & 148 & 156 & $5.13 \%$ \\
\hline
\end{tabular}

\section{conclusion}

This paper proposes a combination of mean generating function and grey prediction model to predict the pesticides amount and in various cities and forecast the pesticide amount of every county in Hainan province as samples. The results show that, The forecast mode of combining the grey GM $(1,1)$ model and the mean generating function can forecast according to the sample data successfully, and has good feasibility.

\section{Acknowledgements}

This work was financially supported by Social development of Hainan province science and technology projects (NO.2015SF35) and National banana industry technology system diseases and insect pest diagnosis software(NO. 00405301).Yong Lin (Chinese Academy of Tropical Agricultural Sciences Institute of Environment and Plant Protection) is Corresponding author, and has equal contribution with Xiu-Hong Zhang (Hainan Normal University) .

\section{References}

[1] YANG Huai-jin, YE Zhi-xiang, XU Cheng-hua, YANG Ying-chun . The GM （1,1） Prediction Model of Pesticide Degradation Optimized by IEA. Journal of Agro-Environment Science.2007,No4.

[2] Wang shuaiyu etc.Beijing 2016 pesticides and YaoXie demand forecasting analysis. Beijing Agriculture. 2016,06:218-219

[3] Deng julong .The basic methodology gray system (version 2)[M] . Wuhan: Huazhong university of science press. 2005.

[4] ZhangXiuHong,LinYong. The application of pesticide usage GIS system base on web service [C]. IEEE ITAIC 2011Vol. 2, 257-258

[5] LIN Yong,ZHANG Xiu-hong,ZHUANG Hong-jie .Construction of the tropical agricultural pesticide database system . AGROCHEINCALS.2011.7 539-542

[6] Bates JM, GrangerC W. The cmibination of forecasts[J]. peration Research Quarterly, 1969, 20(4): 451- 468 